\title{
Division of labor between dendritic cell subsets of the lung
}

\author{
CH GeurtsvanKessel ${ }^{1,2}$ and BN Lambrecht ${ }^{1,3}$
}

\begin{abstract}
Dendritic cells (DCs) are a heterogenous population of antigen-presenting cells, of which conventional DCs and plasmacytoid DCs are the main subsets. Like DC subsets in the central lymphoid organs, DC subsets in the lungs exert specific functions that can be associated with distinct expression of endocytic receptors, cell-surface molecules, and anatomical location within the lung. In recent years, DC populations are increasingly split up into a seemingly endless number of defined sub-populations. We argue that this is not a "stamp-collecting" activity but essential for a deeper understanding of the immune response to pathogens like respiratory viruses or tolerance to harmless antigens. In homeostatic conditions, a fine-tuned balance exists between the various functions of lung DC subsets, which is necessary for maintaining immune homeostasis in the lung. However, infectious or inflammatory conditions can profoundly alter the functions of steady-state DC subsets and recruit inflammatory type DCs to the lung. This might be important for clearing the inflicting pathogenic stimulus, but could at the same time also be involved in causing immune pathology.
\end{abstract}

\section{INTRODUCING DC HETEROGENEITY}

Dendritic cells (DCs) have been described by Banchereau and Steinman ${ }^{1} 25$ years ago, as a population of cells with antigenpresenting capacity found in the spleen and lymph nodes (LNs) of mice. It was initially thought that these cells represented a unique population of cells that could be isolated based on physical characteristics (low density in comparison with other cells, formation of long dendrites), expression of cell-surface molecules such as the integrin $\mathrm{CD} 11 \mathrm{c}$, and the functional characteristic of being able to stimulate naive T-cell proliferation. In the decades following initial discovery, there has been an explosion of papers describing the precise surface phenotype of DCs in various tissues, the ways in which these cells can be generated in vitro, as well as the various ways in which these cells contribute to disease pathogenesis. This has led to a seemingly endless list of papers describing the surface marker expression of DCs. Only recently there have been attempts to try to streamline this information into a general paradigm on how we can subdivide DC subsets that is comprehensive to a novice to the field of DC biology. ${ }^{2,3}$ At least in murine central lymphoid organs, there is a broad division into conventional DCs (cDCs) and plasmacytoid DCs (pDCs). The interested reader is referred to specialized literature on this topic, but broadly speaking, there are at least three subsets of $\mathrm{CD} 11 \mathrm{c}^{\text {hi }} \mathrm{cDCs}\left(\mathrm{CD} 8 \alpha^{+}, \mathrm{CD} 4^{+}\right.$, and $\mathrm{CD} 8 \alpha^{-} \mathrm{CD}^{-}$) and one population of $\mathrm{CD} 11 \mathrm{c}^{\mathrm{int}} \mathrm{pDC}$ in the spleen, whereas in the skin-draining lymph nodes, there are at least five subsets of cells, the extra one representing skin-derived Langerhans cells. ${ }^{4}$ In contrast to cDCs, mouse pDCs uniquely express the endocytic receptor Siglec-H (recognized by the moAb $440 \mathrm{c}),{ }^{5}$ the bone marrow stromal antigen-1 (the latter is recognized by the monoclonal antibodies mPDCA-1 or 120G8), and the B-cell marker B220. pDCs also express high levels of L-selectin and Ly6C, recognized by the Gr-1 antibody (anti-Ly6C/Ly6G), which also marks granulocytes and subsets of monocytes. ${ }^{6}$ In mice, both cDC and pDC subsets can express MHCII, CD80, CD86, CD40, and OX40L and should therefore both be capable of antigen presentation to T cells. ${ }^{7}$ It is, however, clear now that different subsets of DCs perform different tasks, some subsets of cDCs being better at cross-presentation of antigen to CD8 cells on major histo compatibility complex (MHCI) molecules and others better at presenting endocytosed antigen to CD4 T cells on MHCII molecules. ${ }^{8-11}$ These differences might relate to intrinsic differences between DC subsets in-expression of endocytic receptors that target their cargo to well-defined intracellular processing compartments ${ }^{8}$ or to intrinsic differences in the expression levels of MHC-processing machinery proteins in various subsets. ${ }^{10}$

${ }^{1}$ Department of Pulmonary Medicine, Erasmus University Medical Centre, Rotterdam, The Netherlands. ${ }^{2}$ Department of Virology, Erasmus University Medical Centre, Rotterdam, The Netherlands. ${ }^{3}$ Department of Respiratory Diseases, University Hospital Ghent, Ghent, Belgium. Correspondence: BN Lambrecht (bart.lambrecht@ugent.be)

Received 20 July 2008; accepted 3 August 2008; published online 17 September 2008. doi:1038/mi.2008.39 


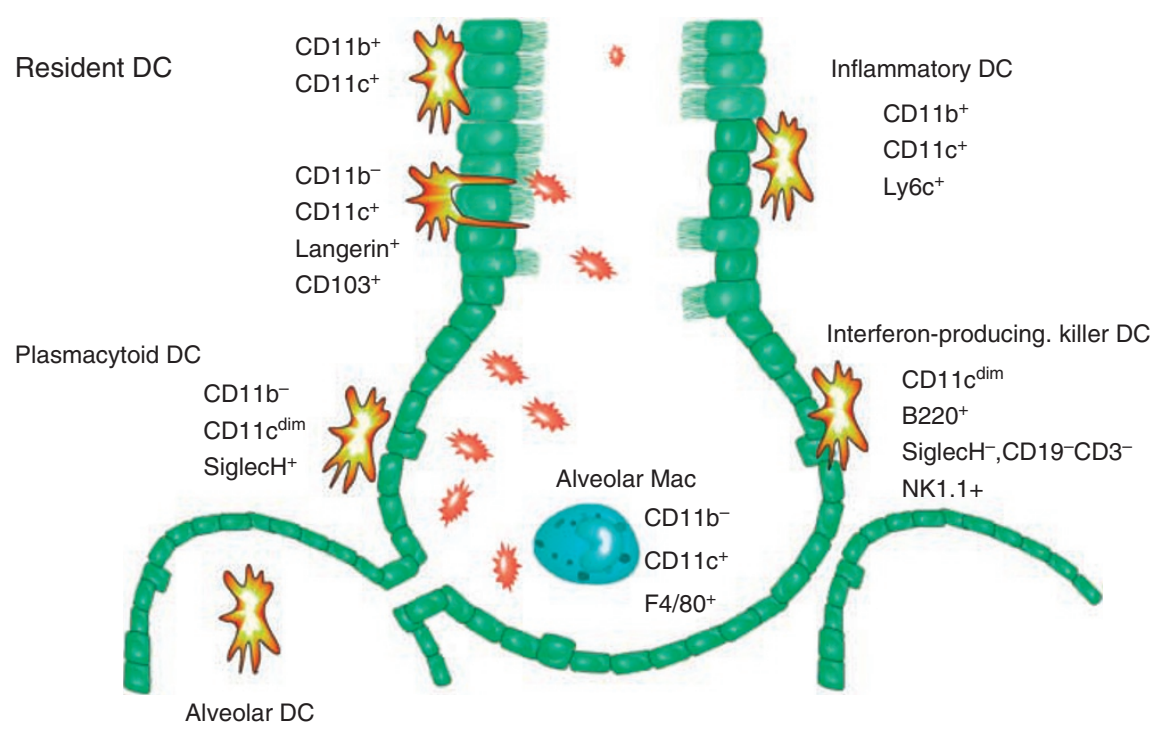

Figure 1 Dendritic cell (DC) subsets in the airways. The conducting airways are composed of airway epithelial cells, which act as a molecular sieve excluding inhaled antigens and pathogens. Two populations of resident DCs are located underneath the epithelial layer. Mucosal CD11b- DCs are situated in the basolateral space and can extend their processes between epithelial cells directly into the airway lumen. This "periscope" function provides a mechanism for continuous immune surveillance of the airway luminal surface. CD11 b+-resident DCs and plasmacytoid DCs (pDCs) are located underneath the basal membrane. In the alveolar space, alveolar DCs and macrophages are located. On inflammatory conditions like respiratory viral infections, an activated population of inflammatory DCs expressing CD11b and Ly6C can be found in the lung tissue as well as the interferon-producing killer DCs (IKDCs).

\section{HETEROGENEITY OF MOUSE LUNG DC SUBSETS}

The subject of lung DC heterogeneity has been appreciated from the starting point when lung DCs were being unraveled in the mouse and rat, and it has since become clear that different DC subsets exert different functions. ${ }^{12-14}$ Here, we will mainly discuss the DC subsets of murine lung (see Figure 1), as this species is the most studied in various animal models of disease. In mouse lungs, DCs are found in most tissue compartments, including the large extrathoracic- and intrathoracic-conducting airways, the lung parenchyma accessible by lung tissue digestion, the alveolar compartment accessible by bronchoalveolar lavage, the pleura, the perivascular space, as well marginating inside the pulmonary lung vessels. ${ }^{15,16}$ Although lung is not a central lymphoid organ, most recent papers now also broadly subdivide lung DCs into cDCs and pDCs, ${ }^{17-19}$ whereas the denominators "lymphoid" and "myeloid" are best abandoned. All cDCs in the mouse express high levels of the integrin CD11c, and these cells can be further subdivided into CD $11 b^{+}$or CD11b ${ }^{-20-22}$ The trachea and large conducting airways have a well-developed network of intraepithelial DCs, even in steady-state conditions. ${ }^{23}$ These cells resemble skin Langerhans cells, and have been shown to express langerin and CD103 but not CD11b. ${ }^{17,20}$ In the submucosa of the conducting airways, $\mathrm{CD}_{103}{ }^{-} \mathrm{CD} 11 \mathrm{~b}^{+} \mathrm{CD} 11 \mathrm{c}^{+}$ cDCs can be found, and these cells are particularly suited for priming and restimulating effector $\mathrm{CD} 4{ }^{+} \mathrm{T}$ cells in the lung. ${ }^{24,25}$ The lung interstitium, which is accessible by enzymatic digestion, also contains $\mathrm{CD}_{1} \mathrm{bb}^{+}$and $\mathrm{CD} 11 \mathrm{~b}^{-} \mathrm{cDC}$ that access the alveolar lumen. ${ }^{21,22,26}$ It should be noted that this population of cells also comprises DCs that line the small intrapulmonary bronchioles, as well as those that line the vessel walls. Intravascular DCs are also highly enriched in the lung capillaries and most authors studying lung DCs try to eliminate this subset before enzymatic digestion of the lung by rinsing the right heart circulation through the pulmonary artery. ${ }^{15} \mathrm{In}$ the nearby alveolar lumen, autofluorescent CD $11 b^{\text {lo }} \mathrm{CD} 11 \mathrm{c}^{\mathrm{hi}}$ alveolar macrophages and alveolar CD $11 b^{\text {hi }}$ CD $11 c^{\text {hi }}$ DCs can be found. One needs to be particularly careful not to confuse $\mathrm{CD} 11 \mathrm{~b}^{\mathrm{lo}} \mathrm{CD} 11 \mathrm{c}^{\text {hi }}$ alveolar macrophages with $\mathrm{CD} 11 \mathrm{~b}^{\text {lo }} \mathrm{cDCs}$, as macrophages can profoundly suppress functional characteristics of lung DCs. ${ }^{27}$ Therefore, protocols that solely rely on purification of lung DCs by CD11c magnetic beads are confounded. We and others commonly use the characteristic autofluorescence of alveolar macrophages to discriminate the two when performing flow- cytometry-based sorting experiments on lung DCs. ${ }^{28-30}$ Lung pDCs in steady state only represent a minor population of CD11 $c^{\text {int }}$ cells that also express bone marrow stromal antigen-1 (recognized by moAb 120G8 and mPDCA-1), Siglec-H, Ly6C, and $\mathrm{B} 220,,^{31-33}$ and can be found in large conducting airways and lung interstitium. ${ }^{21}$

Under inflammatory conditions, things become even more complicated. Inflammatory stimuli such as Toll-like receptor ligands, viral or bacterial infection, or environmental exposure to pollutants such as cigarette smoke or ozone trigger the production of chemokines that attract monocytes and other inflammatory cells to the lungs in a CC-chemokine receptor 2 (CCR2)- and/or CCR5-dependent manner. ${ }^{17,18,34-36}$ These $\mathrm{CCR}^{+}{ }^{+}$monocytes can be the immediate precursors to socalled inflammatory CD $11 b^{\text {hi }}$ CD $11 c^{\text {hi }}$ DCs, which still express high levels of Ly6C as a remnant of their monocytic descent. ${ }^{36}$ Under some conditions, these DCs have been called TIP-DCs for tumour-necrosis factor-producing inducible nitric oxide synthase-producing DCs, ${ }^{37}$ and in some conditions lung iDCs have been shown to produce iducible nitricoxide synthase. ${ }^{36}$ These inflammatory DCs closely resemble the steady-state CD11b ${ }^{+}$ 


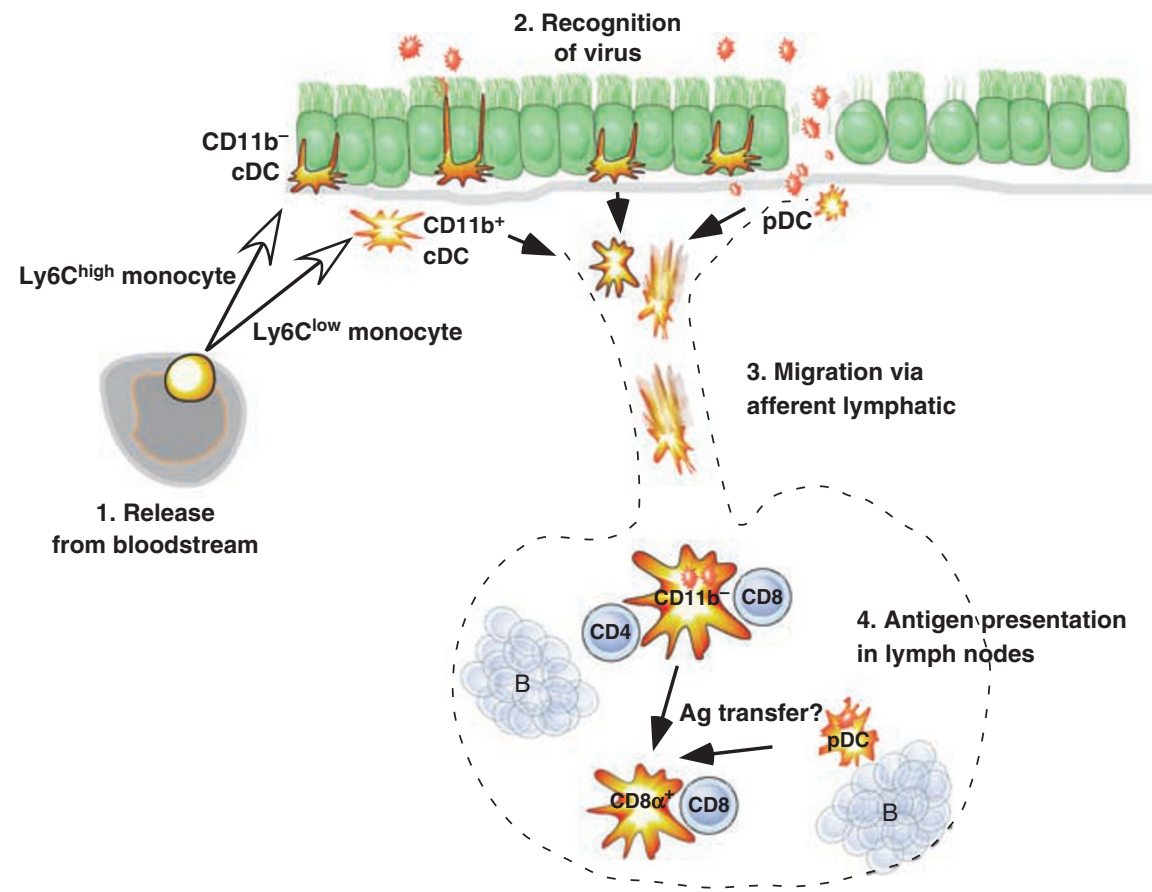

Figure 2 Antigen uptake and presentation by dendritic cells (DCs) in the airways. Dendritic cells are released from the bloodstream and give rise to CD11 $b^{-}$conventional DCs (cDCs; Ly6C high) or CD11b+ ${ }^{+}$CDCs (Ly6Clow). CD11b- DCs can directly recognize viral particles in the airway lumen by sensing with their processes. In contrast, CD11 b+ DCs and plasmacytoid DCs (pDCs) are located underneath the basal membrane and do not pass the epithelial barrier with their processes but pickup antigen that has passed the basal membrane. The different DC populations migrate to the lymph nodes by afferent lymphatics and exert their specific functions. Although the migrated CD11b- DCs can present their antigen to both CD4 and CD8 T cells, the resident lymph node population of CD8 $\alpha^{+}$DCs only presents to CD8 T cells. These resident DCs might obtain their antigen from the migrated CD11 $b^{+}$DCs or pDCs which present antigen less efficiently themselves.

cDCs and therefore some authors have suggested that cDCs "mature" or alter phenotype or even proliferate under inflammatory conditions. ${ }^{19,38}$ A second confounder when studying DC subsets under inflammatory conditions, particularly when high levels of interferon- $\alpha$ (IFN- $\alpha$ ) are being produced during viral infection, is the fact that the bone marrow stromal Ag-1 and B220 is also induced on inflammatory type CD11b ${ }^{+}$DCs, considerably confounding the discrimination between pDCs and $\mathrm{CDCs}$ if the characteristics of size and expression of Siglec- $\mathrm{H}$ is not taken into account (CH GeurtsvanKessel, unpublished). This has led to the misinterpretation that pDCs can differentiate to $\mathrm{CDCs}$ and vice versa or that $\mathrm{pDCs}$ become myeloid like. ${ }^{18,39,40}$ Finally, a recently described population of natural killer (NK) cells with high MHCII and intermediate CD11c was discovered and named IFN-producing killer DCs. ${ }^{41}$ We have recently observed that this subset is also found inside the lungs following inflammatory stimuli ( $\mathrm{CH}$ GeurtsvanKessel, unpublished). As it has a CD11 $\mathrm{c}^{\mathrm{dim}} \mathrm{B} 220^{\mathrm{dim}} \mathrm{MHCII}^{\mathrm{dim}} \mathrm{CD} 1 \mathrm{~b}^{-}, \mathrm{CD}^{-} 9^{-}, \mathrm{CD}^{-}$ phenotype, it is easy to confuse this subset with pDCs if one does not use Siglec-H or a specific NK marker such as NK1.1 or NKp46 to discriminate the two.

\section{ORIGIN OF LUNG DC SUBSETS}

Studies performed by Holt and co-workers ${ }^{42}$ group in the rat and mouse have suggested that the turnover rate of tracheal DCs is in the order of 2-3 days, whereas interstitial lung DCs are much more long lived. ${ }^{22,42}$ Both studies suggested repopulation of DC subsets by a circulating precursor cell derived from the bone marrow. Recent studies performed to elaborate on the origin of the two main $\mathrm{CDC}$ populations, $\mathrm{CD} 11 \mathrm{~b}^{-} \mathrm{CD} 103^{+}$ DCs and $\mathrm{CD} 11 \mathrm{~b}^{+} \mathrm{CD} 103^{-} \mathrm{DCs}$, have demonstrated an origin from distinct circulating monocyte precursors, even in steadystate conditions. ${ }^{43}$ Two distinct monocyte populations have been described in the mouse. A population of Ly6 $\mathrm{C}^{\text {high }} \mathrm{CCR}{ }^{\text {high }}$ (Gr-1 $\left.{ }^{\text {high }}\right)$ monocytes is characterized as the more classical monocytes that readily emigrate to the sites of ongoing inflammation, ${ }^{44,45}$ whereas Ly6C ${ }^{\text {low }}$ CCR $2^{\text {low }}\left(\mathrm{Gr}-1^{\text {low }}\right)$ monocytes expressing high levels of CX3CR1 do not robustly emigrate to many tissues, but they migrate well to lung even in the absence of inflammation. ${ }^{44}$ Jakubzick and colleagues ${ }^{43}$ revealed that $\mathrm{CD} 103^{+} \mathrm{CD} 11 \mathrm{~b}^{-}$preferentially derived from CCR $2^{\text {hi }}$ monocytes, whereas $\mathrm{CD} 11 \mathrm{~b}^{\text {high }} \mathrm{cDCs}$ preferentially arise from CCR2 $2^{\text {lo }}$ monocytes, thus lending proof to the concept that subsets of monocytes recruited to the same tissue undergo differential differentiation pathways within the DC lineage. Others working on lung DC ontogeny have, however, refuted this idea. ${ }^{36,46}$ Studies have been performed in which a spleen-resident precursor of steady-state cDCs was identified. These cells were not monocytes but could nevertheless generate DCs with relatively high efficiency ${ }^{47,48}$ and therefore were named pre-DCs. The equivalent of these pre-DCs were also found in bone marrow and other lymphoid organs ${ }^{47,49}$ implicating that DCs can arise from a precursor that is resident within the lymphoid organ itself and was preprogrammed in the bone 
marrow as a macrophage-DC precursor. ${ }^{2}$ It remains to be determined whether a macrophase-DC precursor or pre-DC population can also be found in the lungs.

\section{FUNCTION OF LUNG DC SUBSETS}

The general paradigm of DC function states that DCs reside in an immature state in the periphery of the lung, where they sample the inhaled air for incoming antigen (see Figure 2). On triggering the antigen-recognition receptors such as Toll-like receptors, the cells migrate through the afferent lymphatics to the T-cell area where they select and activate naive T cells and differentiate them into either tolerizing Treg cells or cytokinesecreting $\mathrm{T}$ effector cells. Considerable progress has recently been made to suggest that different lung DC subsets perform various aspects of this paradigm differentially. The conducting airways are lined with a mucociliary blanket and composed of airway epithelial cells that are connected by tight junctions and zonula adherens. The epithelial-cell layer acts as a molecular sieve that excludes inhaled antigens and pathogens based on their molecular weight. Intraepithelial CD11b ${ }^{-}$DCs are situated in the basolateral space, only separated from the inhaled air by the epithelium tight-junction barrier. In the airways, as well as in the gut, DCs can extend their processes between epithelial cells directly into the airway lumen. This "periscope" function is constitutively active in the airway mucosal DC population, providing a mechanism for continuous immune surveillance of the airway luminal surface. ${ }^{50-52}$ At least in mouse lungs, intraepithelial CD $103^{+}$DCs express the tight-junction proteins claudin-1, claudin-7, and zonula-2, which form tight junctions with airway epithelial cells, thereby explaining how DCs can sample the content of the airway lumen while keeping the epithelium barrier function intact. ${ }^{20}$

Following sampling of airway luminal contents, there is transport of antigen to the mediastinal nodes. When large fluorescently labeled antigens are injected into the lungs of mice, different subsets of DCs become antigen positive in the draining mediastinal lymph nodes as early as $12 \mathrm{~h}$ after antigen administration. ${ }^{21,30}$ There are several mechanisms by which the inhaled antigen could reach the lymph nodes (for more detailed discussion on this topic see ref. 21). Most of the experimental evidence suggests that antigen is taken up by DCs in the lung, which then migrates in a CCR7- and CCR8-dependent manner to the draining mediastinal lymph nodes, which is analogous to the directed migration of skin DCs. ${ }^{21,53}$ It is still unclear exactly where and by which DC subset the inhaled antigen is sampled from the airways, as both mucosal cDCs that line the conducting airways and cDCs that are situated in the alveolar wall are exposed to inhaled antigens, and have the potential to migrate to the lymph nodes. ${ }^{21,26,33,54}$ Similarly, only one study briefly showed that pDCs also might migrate from the periphery of the lung to the mediastinal node during viral infection. ${ }^{19}$

In addition to cell-mediated transport, the tight-junction barrier might act as a molecular sieve that allows the passive leakage of smaller antigens into the afferent lymph vessels. Antigen sampled in such a passive manner might gain access to resident DCs in the draining lymph node, such as CD8 $\alpha^{+}$
DCs or pDCs. ${ }^{33}$ Although there is indeed evidence for the passive transfer of fluorescently labeled molecules to the mediastinal lymph nodes, no studies to date have demonstrated that antigen that reaches the resident DCs in this way can result in the induction of T-cell division. ${ }^{21,30,33}$ The final and the most important aspect of DC function is the capacity of these cells to program T-cell responses. Regarding functional differences in DC populations from airway mucosa vs. parenchymal tissue, von Garnier et al. ${ }^{21,22}$ demonstrated that airway mucosal cDCs were more endocytic and presented peptide to naive $\mathrm{CD} 4{ }^{+} \mathrm{T}$ cells more efficiently than their lung counterparts. However, only DCs matured in vitro or after migration to lymph nodes in vivo could present whole protein to $\mathrm{T}$ cells and induce $\mathrm{T}$-cell activation. This finding indicates a mechanism in which DC function is regulated at the level of protein processing, rather than peptide loading.

It has been shown that inhalation of harmless antigen leads to T-cell unresponsiveness in the process that is accompanied by DC-driven T-cell proliferation and formation of Tregs. ${ }^{55,56}$ Whether this is a function of particular lung DC subsets still remains a matter of debate. We and others have shown that inhalation tolerance clearly depends on the presence of pDCs that take up inhaled harmless antigen and leads to the formation of Treg cells. ${ }^{31-33}$ Others have shown that this process also required CDCs that depend on CCR7 for migration to the mediastinal nodes. ${ }^{25,53}$ In this regard, del Rio et al. ${ }^{25}$ suggested that $\mathrm{CD}_{103}{ }^{+} \mathrm{CD}_{11 \mathrm{~b}^{-}} \mathrm{cDCs}$ were specialized in cross-tolerizing CD8 responses, whereas $\mathrm{CD} 11 \mathrm{bhi}$ cDCs were specialized in tolerizing CD4 T-cell responses to inhaled harmless antigen.

Antigens encountered in the lung by DCs as part of a respiratory pathogen or model protein antigens administered together with different Toll-like receptor ligands can induce different types of effector T-helper responses. ${ }^{57} \mathrm{~A}$ detailed description on how T-cell effector responses are programmed is beyond the scope of this study, but suffice it to say that cytokines and costimulatory molecules are the most important factors that will determine the outcome of a pulmonary immune response driven by lung DCs. Stumbles ${ }^{58}$ and Dodge ${ }^{59}$ have demonstrated that lung DCs are biased toward inducing Th2 responses, through production of interleukin-6 (IL-6), but it is not yet clear whether this property could be linked to the distinct properties of a particular CDC subset in a particular location. In any case, depending on the type of inflammatory stimulus, lung DCs can induce cytotoxic T-lymphocyte responses and Th1, Th2, or Th17 Th effector responses. In the coming paragraphs we will illustrate how a better understanding of DC subsets of the lungs has provided novel insights into how effector T-cell responses are induced in the lung.

\section{SUBSETS IN RESPIRATORY VIRAL INFECTIONS Influenza}

Influenza virus causes upper and lower respiratory tract infection, of which clinical outcome is mainly determined by the way in which the innate and adaptive immune system initially recognizes and deals with the replicating virus. ${ }^{17,18,60-62}$ On influenza virus infection, immediate innate defense mechanisms come into play that lead to high-level production of type I IFNs by infected 
lung epithelial cells, alveolar macrophages, and natural IFN-producing cells (also known as pre-pDCs). Innate chemokines promote the recruitment of neutrophils, NK cells, and DCs. ${ }^{1,63}$

Several investigators in the past have studied the involvement of antigen-presenting cells such as DCs, macrophages, and B cells in mediating protective immunity to influenza virus. ${ }^{64}$ It was shown that a mouse-adapted strain of influenza virus induced the in vivo maturation of $\mathrm{CD} 11 \mathrm{c}^{+}$DCs in the lung ${ }^{65}$ and their migration to the mediastinal LNs (MLNs). ${ }^{66-68}$ More recently, our group has demonstrated that the predominant

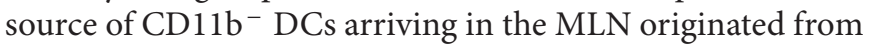
the network of highly dendritic-shaped $\mathrm{CD}_{103}{ }^{+}$intraepithelial DCs that lines the large conducting airways. ${ }^{17,20,23}$ The disappearance of this $\mathrm{CD} 11 \mathrm{~b}^{-}$population from the trachea was accompanied by a new influx of $\mathrm{CD} 11 \mathrm{~b}^{+} \mathrm{DCs}$ into the trachea and interstitium. These freshly recruited DCs most likely represent inflammatory-type DCs, ${ }^{48,69}$ a highly activated subset of cDCs found in the lungs on respiratory infection. ${ }^{36}$ We have data to suggest that in influenza virus infection, these inflammatory DCs express B220 and 120G8 in addition to high levels of $\mathrm{CD} 11 \mathrm{c}$ and $\mathrm{CD} 11 \mathrm{~b}$, considerably confusing the separation from pDC subsets if multi-parameter flow cytometry is not used (see above, CH GeurtsvanKessel, unpublished).

Once arrived in the MLN, both CD11 $\mathrm{b}^{-} \mathrm{CD} 8 \mathrm{a}^{+}$-resident DCs and $\mathrm{CD}_{11 \mathrm{~b}^{-}}$and $\mathrm{CD} 11 \mathrm{~b}^{+}$lung-derived migratory DCs upregulate costimulatory molecules, and thus acquire the potential to present antigen. There is however a clear division of labor. The

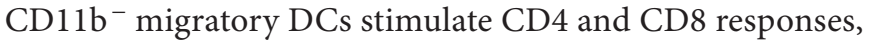
whereas $\mathrm{CD} 11 \mathrm{~b}^{+}$DCs are very poor at antigen presentation. This suggests that on influenza virus infection, processing for and presentation on both MHCI and MHCII molecules can occur in a single cell $\mathrm{CD}_{11 \mathrm{~b}^{-}}$population in vivo in the lung, in contrast to what previously has been shown for spleen or lymph node. ${ }^{8-10,70}$ Consequently, selective lung depletion of these $\mathrm{CD} 11 \mathrm{~b}^{-} \mathrm{cDCs}$ in mice carrying the diphteria toxin receptor under the langerin promoter led to a severe reduction in virus- specific CD8 T cells (identified through tetramer staining) and a delay in viral clearance from the lung. ${ }^{17}$ There is an additional population of $\mathrm{CD} 8 \alpha^{+} \mathrm{CD} 11 b^{-}$lymph node-resident cDCs that acquired antigen from another migratory cells and presents exclusively to CD $8 \mathrm{~T}$ cells, as previously shown by others. ${ }^{71-73}$ Probably, the most important source for viral antigen to resident CD11 $\mathrm{b}^{-} \mathrm{CD} 8 \mathrm{a}^{+}$DCs in MLN are the CD11b ${ }^{+}$DCs and $\mathrm{pDCs}$ that contain massive amounts of viral antigen but do not seem to present very well in the node. ${ }^{17}$

If $\mathrm{CD}_{11} \mathrm{~b}^{+} \mathrm{cDCs}$ and inflammatory DCs do not present antigen efficiently in the MLN, then what will be their contribution? First, lung CD11b ${ }^{+}$DCs have been shown to massively produce inflammatory chemokines and might therefore be crucial in attracting effector CD4 and CD8 cells that have been generated in the LN back to the lung and trachea, where they would mediate effector function. ${ }^{74}$ Recently, Legge and co-workers ${ }^{18}$ also suggested that these inflammatory type DCs might indeed be crucial for boosting effector CD8 responses in the lung, rather than priming of CD8 responses in naive cells. Second, CD11b ${ }^{+}$DCs might have direct innate antivi- ral activity by producing tumor-necrosis factor- and iducible nitricoxide synthase-dependent nitric oxide, analogous to the situation seen with Listera monocytogenes infection, ${ }^{37,75}$ and thus might also contribute to the features of severe influenza such as lung damage and weight loss. ${ }^{36}$ Third, recruited CD11 $\mathrm{b}^{+}$ DCs might also stimulate the innate antiviral activity through cross -talk with NK cells.

The precise role of $\mathrm{pDCs}$ in influenza virus infection remains undetermined from carefully studying the literature. Although they are main producers of type 1 IFNs during both in vivo and in vitro influenza virus infection, ${ }^{76,77}$ this role seems to be taken over by other (epithelial and alveolar macrophages) cells in case pDCs are depleted. ${ }^{17,63}$ Migration of pDCs to MLNs on infection or inflammation has been demonstrated ${ }^{17,19,33}$ but their contribution to antigen presentation at this site seems to be negligible in vivo, as depletion of pDCs through specific antibodies before infection did not affect viral clearance or induction of virus-specific CD8 T cells. Recently, however, it was shown that human pDCs did have the potential to cross-present viral influenza antigen to CD8 $\mathrm{T}$ cells in vitro. ${ }^{78}$ It could still be that we failed to detect antigen-presenting cell function in lymph node pDCs because we studied the wrong compartment at a wrong point in time. Overall, it seems that on influenza virus infection, lung pDCs (besides the cDCs) mainly accumulate at the site of infection and do not direct their interest to the MLN. ${ }^{18}$ It remains to be seen whether lung (as opposed to MLN) pDCs present viral-derived antigen or not to CD4 or CD8 T cells. Another possibility would be that pDCs contribute to antibody production by B cells. ${ }^{17,79}$

\section{RSV and other paramyxoviridae}

Respiratory syncytial virus (RSV) is a respiratory pathogen causing bronchiolitis in infants and severe lower respiratory tract infection and pneumonia in elderly. In addition, RSV infection at a young age is a risk factor for asthma development in childhood. ${ }^{80,81}$ Severe RSV disease is associated with excessive pulmonary inflammation, primarily due to host immune responses, rather than a direct cytopathic effect of the virus. ${ }^{82}$

Respiratory syncytial virus increases the number of both $\mathrm{cDCs}$ and $\mathrm{pDCs}$ in the lung and MLN throughout the inflammatory phase of infection, but in contrast to influenza virus the number of pDCs remain elevated during the resolution phase of infection. ${ }^{83}$ IFN- $\alpha$ production by pDCs in response to RSV infection has only been demonstrated in vitro. ${ }^{76,83} \mathrm{~A}$ study by Jewell et al. ${ }^{76}$ furthermore demonstrated that in RSV infection the infected respiratory epithelium was a major source of IFN$\alpha / \beta$. Despite the minor role of $\mathrm{pDCs}$ in producing IFN during RSV infection, studies in which pDCs have been depleted demonstrated a decreased viral clearance and an exacerbation of immune-mediated pathology ${ }^{83,84}$ As in asthmatic conditions, the balance of $\mathrm{CDCs}$ and pDCs in lung tissue has an important function in outcome of RSV infection. ${ }^{33,85}$ Depletion of pDCs mainly leads to enhanced airway hyperreactivity, mucus production, and inflammation; and increasing numbers of $\mathrm{pDCs}$ by adoptive transfer have a protective impact on the infection..$^{85}$ In contrast to influenza virus infection, removal of pDCs did have 
an effect on viral clearance, possibly due to effects of Th2-driven immunopathology on the potential to generate CD8 cytotoxic $\mathrm{T}$ cells. Sendai virus is another paramyxovirus that has been studied as a model of RSV in the mouse. Also in this model, infection leads to Th2-mediated immunopathology characterized by mucus cell hyperplasia. Strikingly, in this model, virus infection leads to upregulation of the high the affinity receptor for immunoglobulin E on lung cDCs or inflammatory DCs, a finding suggesting that virus infection might promote Th2 responses through modification of handling of $\mathrm{Ag}$ by DCs. ${ }^{86}$

\section{SUBSETS IN ASTHMA}

Allergic asthma is a chronic inflammatory disease of the airways driven by Th 2 cells that produce cytokines such as IL-4, IL-5, and IL-13 that mediate pathology. Despite the fact that most inhaled antigens are transported to the lymph node by lung-derived DCs, the usual outcome following the inhalation of harmless protein antigen is the induction of tolerance. When the model antigen ovalbumin is given into the airways of naive mice, it renders the mice tolerant to a subsequent immunization with ovalbumin in alum adjuvant, and effectively inhibits the development of allergic airway inflammation, in a process requiring DC migration to the LNs, T-cell division, and expansion of antiinflammatory Treg cells. ${ }^{30,53,87,88}$ It is not clear at present which particular subset of $\mathrm{CDCs}$ might promote tolerance. In the gut, it was suggested that $\mathrm{CD} 103^{+} \mathrm{cDCs}$ promote tolerance by promoting Treg cell formation, but this remains to be shown for the $\mathrm{CD} 11 \mathrm{~b}^{-} \mathrm{CD} 103^{+}$lung counterpart. ${ }^{89}$ One possible explanation is that in the naive mouse the harmless antigen encountered by lung-derived cDCs cannot fully activate the cDCs or fails to recruit new inflammatory type DCs to induce an effective T-cell response. ${ }^{56}$ Alternatively, partially mature DCs could stimulate the induction of IL-10- and/or transforming growth factor- $\beta$ producing regulatory T cells, in an IL-10- and/or inducible Tcell costimulator ligand-dependent manner. ${ }^{56,87,90,91}$

The process of inhalational tolerance is also influenced by pDCs. Following inhalation of ovalbumin, mediastinal pDCs internalize ovalbumin, but in contrast to cDCs, they do not present the antigen in an immunogenic manner to $\mathrm{CD} 4^{+} \mathrm{T}$ cells. ${ }^{32,33}$ However, functional evidence that these cells do influence pulmonary adaptive immunity came from experiments in which pDCs were depleted using antibodies. In pDC-depleted animals, inhalation tolerance was abolished, whereas following adoptive transfer of FMS-related tyrosine kinase 3-cultured bone-marrow-derived pDCs, tolerance was induced. ${ }^{33}$ How exactly the depletion of pDCs results in Th2-cell sensitization is still unresolved, but in vitro and in vivo data suggest that pDCs directly suppress the potential of lung-derived cDCs to generate effector T cells. ${ }^{33,92}$ Both in vitro and in vivo studies have clearly shown that $\mathrm{pDCs}$ can also stimulate the induction of regulatory $\mathrm{T}$ cells, possibly in an inducible T-cell costimulator ligand-dependent manner. ${ }^{33,92-95}$ Once induced, regulatory $\mathrm{T}$ cells could also induce the production of the tryptophan-catabolizing enzyme indoleamine 2,3-dioxygenase through reverse signaling in pDCs. ${ }^{96}$

If the usual outcome of inhalation of antigen is the induction of tolerance by partially mature lung-derived DCs, it follows that some degree of $\mathrm{cDC}$ activation or recruitment of inflammatory type DCs could be sufficient to break tolerance and promote Th2-cell development. Studies by Kim Bottomly and co-worker's ${ }^{97}$ have elegantly shown that triggering of TLR4 on lung-derived cDCs by low doses of lipopolysaccharide is sufficient to break inhalation tolerance and promote Th2-cell development through a myeloid differentiation primary-response gene 88 (MyD88)-dependent pathway. This is clinically relevant as most of the inhaled allergens, such as cockroach and house dust mite allergens, are contaminated with lipopolysaccharide and peptidoglycans, and there is a clear relationship between the degree of LPS coexposure and the risk of sensitization. ${ }^{97}$ Very recently, it was also shown that the generation of Th2 immunity in the lung is a function of a poorly defined $\mathrm{CDC}$ subset that selectively expresses the receptor for stem cell factor, called c-Kit. The expression and triggering of c-Kit receptor by cell-bound stem cell factor on this DC subset promoted Th2 responses in a process requiring IL-6 secretion. ${ }^{59,98}$

$\mathrm{cDCs}$ and $\mathrm{pDCs}$ not only have a function in the primary immune response to inhaled allergens, but also have a crucial role during the effector phase of asthma, and thus might constitute good targets for the development of new therapies. ${ }^{99-103}$ The number of CD11 $\mathrm{b}^{+}$inflammatory DCs is increased in the conducting airways, lung interstitium, and mediastinal LN of sensitized and challenged mice during the acute phase of the response. ${ }^{104,105}$ In asthmatics, allergen challenge leads to an accumulation of myeloid DCs, but not pDCs, in the airways, concomitantly with a reduction in circulating $\mathrm{CD} 11 \mathrm{c}^{+} \mathrm{DCs}$, indicating that these cells are recruited from the bloodstream in response to allergen challenge. ${ }^{106} \mathrm{~A}$ functional role for DCs in the secondary immune response was further supported by the fact that their depletion at the time of allergen challenge abrogated all the characteristic features of asthma. ${ }^{23,24}$ The defect was restored by the intratracheal administration of bone-marrow-derived granulocyte macrophase colony-stimulating factor (GM-CSF) derived CD $11 b^{+}$DCs, which most closely resemble the monocyte-derived inflammatory DCs that are recruited on allergen challenge. The reason why CD11 $\mathrm{b}^{+} \mathrm{DCs}$ might be so crucial for asthma is currently unknown. Following allergen challenge, DCs upregulate the expression of several costimulatory molecules that might be involved in T-cell activation. ${ }^{107,108}$ Moreover, in allergen-challenged mice, $\mathrm{CD} 11 \mathrm{~b}^{+} \mathrm{DCs}$ might also be a prominent source of the Th2 cell-attracting inflammatory chemokines CCL17 and CCL22, and of eosinophil-selective chemokines. ${ }^{74,109}$ A number of cytokines and innate immune factor regulate the production of these chemokines. For instance, epithelial cell-derived thymic stromal lymphopoietin (TSLP)can increase CCL17 and CCL22 production by myeloid DCs and might therefore contribute to enhanced inflammation. ${ }^{110}$ For a detailed discussion on epithelial influences on DC function in asthma, we refer to a recent paper on this topic. ${ }^{99}$

\section{CONCLUSION}

The examples described above clearly illustrate the complexity in the field of DC biology in the lung. We however believe that a full understanding of the function of these cells during homeo- 
stasis and immune responses will never be attained if we do not take DC heterogeneity into account. The biggest challenge will be to integrate this knowledge to the human pulmonary immune responses, as this might have big implications for understanding allergic and microbial pathogenesis in the human situation.

\section{DISCLOSURE}

The authors declared no conflict of interest.

() 2008 Society for Mucosal Immunology

\section{REFERENCES}

1. Banchereau, J. \& Steinman, R.M. Dendritic cells and the control of immunity. Nature 392, 245-252 (1998).

2. Naik, S.H. Demystifying the development of dendritic cell subtypes, a little. Immunol. Cell Biol. 86, 439-452 (2008).

3. Shortman, K. \& Liu, Y.J. Mouse and human dendritic cell subtypes. Nat. Rev. Immunol. 2, 151-161 (2002).

4. Henri, S. et al. The dendritic cell populations of mouse lymph nodes. J. Immunol. 167, 741-748 (2001).

5. Blasius, A. et al. A cell-surface molecule selectively expressed on murine natural interferon-producing cells that blocks secretion of interferonalpha. Blood 103, 4201-4206 (2004).

6. Nakano, H., Yanagita, M. \& Gunn, M.D. CD11C(+)B220(+)Gr-1(+) cells in mouse lymph nodes and spleen display characteristics of plasmacytoid dendritic cells. J. Exp. Med. 194, 1171-1178 (2001)

7. Boonstra, A. et al. Flexibility of mouse classical and plasmacytoidderived dendritic cells in directing Thelper type 1 and 2 cell development: dependency on antigen dose and differential toll-like receptor ligation. J. Exp. Med. 197, 101-109 (2003).

8. Burgdorf, S., Kautz, A., Bohnert, V., Knolle, P.A. \& Kurts, C. Distinct pathways of antigen uptake and intracellular routing in CD4 and CD8 T cell activation. Science 316, 612-616 (2007).

9. Burgdorf, S., Scholz, C., Kautz, A., Tampe, R. \& Kurts, C. Spatial and mechanistic separation of cross-presentation and endogenous antigen presentation. Nat. Immunol. 9, 558-566 (2008).

10. Dudziak, D. et al. Differential antigen processing by dendritic cell subsets in vivo. Science 315, 107-111 (2007).

11. Sancho, D. et al. Tumor therapy in mice via antigen targeting to a novel, DC-restricted C-type lectin. J. Clin. Invest. 118, 2098-2110 (2008).

12. Xia, W., Schneeberger, E., Mc Carthy, K.M. \& Kradin, R.L. Accessory cells of the lung II: la+ pulmonary dendritic cells display cell surface antigen heterogeneity. Am. J. Respir. Cell Mol. Biol. 5, 276-283 (1991).

13. Kradin, R.L., Xia, W., Mc Carthy, K.M. \& Schneeberger, E.E. FcR ${ }^{+/-}$ subsets of la $\mathrm{a}^{+}$pulmonary dendritic cells in the rat display differences in their abilities to provide accessory co-stimulation for naive $\left(\mathrm{Ox}-22^{+}\right)$and sensitized (Ox-22-) T cells. Am. J. Pathol. 142, 811-819 (1993).

14. Schon-Hegrad, M.A., Oliver, J., McMenamin, P.G. \& Holt, P.G. Studies on the density, distribution and surface phenotype of intraepithelial class II major histocompatibility complex antigen (la)-bearing dendritic cells (DC) in the conducting airways. J. Exp. Med. 173, 1345-1356 (1991).

15. Suda, T., McCarthy, K., Vu, Q., McCormack, J. \& Schneeberger, E.E. Dendritic cell precursors are enriched in the vascular compartment of the lung. Am. J. Respir. Cell Mol. Biol. 19, 728-737 (1998).

16. Sertl, K. et al. Dendritic cells with antigen-presenting capability reside in airway epithelium, lung parenchyma, and visceral pleura. J. Exp. Med. 163, 436-451 (1986).

17. GeurtsvanKessel, C.H. et al. Clearance of influenza virus from the lung depends on migratory langerin+CD11b - but not plasmacytoid dendritic cells. J. Exp. Med. 205, 1621-1634 (2008).

18. McGill, J., Van Rooijen, N. \& Legge, K.L. Protective influenza-specific CD8 T cell responses require interactions with dendritic cells in the lungs. J. Exp. Med. 205, 1635-1646 (2008).

19. Grayson, M.H. et al. Controls for lung dendritic cell maturation and migration during respiratory viral infection. J. Immunol. 179, 1438-1448 (2007).

20. Sung, S.S. et al. A major lung CD103 (alphaE)-beta7 integrin-positive epithelial dendritic cell population expressing Langerin and tight junction proteins. J. Immunol. 176, 2161-2172 (2006).
21. Wikstrom, M.E. \& Stumbles, P.A. Mouse respiratory tract dendritic cell subsets and the immunological fate of inhaled antigens. Immunol. Cell Biol. 85, 182-188 (2007).

22. von Garnier, C. et al. Anatomical location determines the distribution and function of dendritic cells and other APCs in the respiratory tract. J. Immunol. 175, 1609-1618 (2005).

23. Lambrecht, B.N., Salomon, B., Klatzmann, D. \& Pauwels, R.A. Dendritic cells are required for the development of chronic eosinophilic airway inflammation in response to inhaled antigen in sensitized mice. J. Immunol. 160, 4090-4097 (1998).

24. van Rijt, L.S. et al. In vivo depletion of lung CD11C+ dendritic cells during allergen challenge abrogates the characteristic features of asthma. J. Exp. Med. 201, 981-991 (2005).

25. del Rio, M.L., Rodriguez-Barbosa, J.I., Kremmer, E. \& Forster, R. CD103- and CD103+ bronchial lymph node dendritic cells are specialized in presenting and cross-presenting innocuous antigen to CD4+ and CD8+ T cells. J. Immunol. 178, 6861-6866 (2007).

26. Cleret, A. et al. Lung dendritic cells rapidly mediate anthrax spore entry through the pulmonary route. J. Immunol. 178, 7994-8001 (2007).

27. Holt, P.G., Schon-Hegrad, M.A. \& Oliver, J. MHC class II antigen-bearing dendritic cells in pulmonary tissues of the rat (regulation of antigen presentation activity by endogenous macrophage populations). J. Exp. Med. 167, 262-274 (1988).

28. Vermaelen, K. \& Pauwels, R. Accurate and simple discrimination of mouse pulmonary dendritic cell and macrophage populations by flow cytometry: methodology and new insights. Cytometry A 61A, 170-177 (2004).

29. van Rijt, L.S. et al. A rapid flow cytometric method for determining the cellular composition of bronchoalveolar lavage fluid cells in mouse models of asthma. J. Immunol. Methods 288, 111-121 (2004).

30. Vermaelen, K.Y., Carro-Muino, I., Lambrecht, B.N. \& Pauwels, R.A. Specific migratory dendritic cells rapidly transport antigen from the airways to the thoracic lymph nodes. J. Exp. Med. 193, 51-60 (2001).

31. Xanthou, G. et al. Osteopontin has a crucial role in allergic airway disease through regulation of dendritic cell subsets. Nat. Med. 13, 570-578 (2007).

32. Oriss, T.B. et al. Dynamics of dendritic cell phenotype and interactions with CD4+ T cells in airway inflammation and tolerance. J. Immunol. 174, 854-863 (2005).

33. De Heer, H.J. et al. Essential role of lung plasmacytoid dendritic cells in preventing asthmatic reactions to harmless inhaled antigen. J. Exp. Med. 200, 89-98 (2004).

34. Robays, L.J. et al. Chemokine receptor CCR2 but not CCR5 or CCR6 mediates the increase in pulmonary dendritic cells during allergic airway inflammation. J. Immunol. 178, 5305-5311 (2007).

35. Osterholzer, J.J. et al. CCR2 and CCR6, but not endothelial selectins, mediate the accumulation of immature dendritic cells within the lungs of mice in response to particulate antigen. J. Immunol. 175, 874-883 (2005).

36. Lin, K.L., Suzuki, Y., Nakano, H., Ramsburg, E. \& Gunn, M.D. CCR2+ monocyte-derived dendritic cells and exudate macrophages produce influenza-induced pulmonary immune pathology and mortality. J. Immunol. 180, 2562-2572 (2008).

37. Serbina, N.V., Salazar-Mather, T.P., Biron, C.A., Kuziel, W.A. \& Pamer, E.G. TNF/iNOS-producing dendritic cells mediate innate immune defense against bacterial infection. Immunity 19, 59-70 (2003).

38. Wang, H. et al. Local CD11 C+ MHC class II- precursors generate lung dendritic cells during respiratory viral infection, but are depleted in the process. J. Immunol. 177, 2536-2542 (2006).

39. Zuniga, E.I., McGavern, D.B., Pruneda-Paz, J.L., Teng, C. \& Oldstone, M.B. Bone marrow plasmacytoid dendritic cells can differentiate into myeloid dendritic cells upon virus infection. Nat. Immunol. 5, 1227-1234 (2004).

40. Comeau, M.R., Van Der Vuurst De Vries, A.R., Maliszewski, C.R. \& Galibert, L. CD123(bright) plasmacytoid predendritic cells: progenitors undergoing cell fate conversion? J. Immunol. 169, 75-83 (2002).

41. Taieb, J. et al. A novel dendritic cell subset involved in tumor immunosurveillance. Nat. Med. 12, 214-219 (2006).

42. Holt, P.G., Haining, S., Nelson, D.J. \& Sedgwick, J.D. Origin and steadystate turnover of class II MHC-bearing dendritic cells in the epithelium of the conducting airways. J. Immunol. 153, 256-261 (1994). 
43. Jakubzick, C. et al. Blood monocyte subsets differentially give rise to CD103+ and CD103 - pulmonary dendritic cell populations. J. Immunol. 180, 3019-3027 (2008).

44. Geissmann, F., Jung, S. \& Littman, D.R. Blood monocytes consist of two principal subsets with distinct migratory properties. Immunity 19, 71-82 (2003).

45. Le Borgne, M. et al. Dendritic cells rapidly recruited into epithelial tissues via CCR6/CCL20 are responsible for CD8+ T cell crosspriming in vivo. Immunity 24, 191-201 (2006).

46. Landsman, L., Varol, C. \& Jung, S. Distinct differentiation potential of blood monocyte subsets in the lung. J. Immunol. 178, 2000-2007 (2007).

47. Diao, J. et al. In situ replication of immediate dendritic cell (DC) precursors contributes to conventional DC homeostasis in lymphoid tissue. J. Immunol. 176, 7196-7206 (2006).

48. Naik, S.H. et al. Development of plasmacytoid and conventional dendritic cell subtypes from single precursor cells derived in vitro and in vivo. Nat. Immunol. 8, 1217-1226 (2007).

49. Diao, J., Winter, E., Chen, W., Cantin, C. \& Cattral, M.S. Characterization of distinct conventional and plasmacytoid dendritic cell-committed precursors in murine bone marrow. J. Immunol. 173, 1826-1833 (2004).

50. Jahnsen, F.L. et al. Accelerated antigen sampling and transport by airway mucosal dendritic cells following inhalation of a bacterial stimulus. J. Immunol. 177, 5861-5867 (2006).

51. Asokananthan, N. et al. House dust mite allergens induce proinflammatory cytokines from respiratory epithelial cells: the cysteine protease allergen, Der $\mathrm{p} 1$, activates protease-activated receptor (PAR)2 and inactivates PAR-1. J. Immunol. 169, 4572-4578 (2002).

52. Chieppa, M., Rescigno, M., Huang, A.Y. \& Germain, R.N. Dynamic imaging of dendritic cell extension into the small bowel lumen in response to epithelial cell TLR engagement. J. Exp. Med. 203, 28412852 (2006).

53. Hintzen, G. et al. Induction of tolerance to innocuous inhaled antigen relies on a CCR7-dependent dendritic cell-mediated antigen transport to the bronchial lymph node. J. Immunol. 177, 7346-7354 (2006).

54. von Garnier, C. et al. Allergic airways disease develops after an increase in allergen capture and processing in the airway mucosa. J. Immunol. 179, 5748-5759 (2007).

55. Stock, P. et al. Induction of $\mathrm{T}$ helper type 1 -like regulatory cells that express Foxp3 and protect against airway hyper-reactivity. Nat. Immunol. 5, 1149-1156 (2004).

56. Akbari, O. et al. Antigen-specific regulatory T cells develop via the ICOSICOS-ligand pathway and inhibit allergen-induced airway hyperreactivity. Nat. Med. 8, 1024-1032 (2002).

57. Kuipers, H. et al. Lipopolysaccharide-induced suppression of airway Th2 responses does not require IL-12 production by dendritic cells. J. Immunol. 171, 3645-3654 (2003).

58. Stumbles, P.A. et al. Resting respiratory tract dendritic cells preferentially stimulate Thelper cell type 2 (Th2) responses and require obligatory cytokine signals for induction of Th1 immunity. J. Exp. Med. 188, 20192031 (1998).

59. Dodge, I.L., Carr, M.W., Cernadas, M. \& Brenner, M.B. IL-6 production by pulmonary dendritic cells impedes Th1 immune responses. J. Immunol. 170, 4457-4464 (2003).

60. Eichelberger, M., Allan, W., Zijlstra, M., Jaenisch, R. \& Doherty, P.C. Clearance of influenza virus respiratory infection in mice lacking class I major histocompatibility complex-restricted CD8+ T cells. J. Exp. Med. 174, 875-880 (1991).

61. Pichlmair, A. et al. RIG-I-mediated antiviral responses to single-stranded RNA bearing 5'-phosphates. Science 314, 997-1001 (2006).

62. Belz, G.T. et al. Bone marrow-derived cells expand memory CD8+ T cells in response to viral infections of the lung and skin. Eur. J. Immunol. 36, 327-335 (2006).

63. Kumagai, Y. et al. Alveolar macrophages are the primary interferon-alpha producer in pulmonary infection with RNA viruses. Immunity 27, 240252 (2007).

64. Hamilton-Easton, A. \& Eichelberger, M. Virus-specific antigen presentation by different subsets of cells from lung and mediastinal lymph node tissues of influenza virus-infected mice. J. Virol. 69, 63596366 (1995).

65. Yamamoto, N. et al. Dendritic cells are associated with augmentation of antigen sensitization by influenza A virus infection in mice. Eur. J. Immunol. 30, 316-326 (2000).
66. Brimnes, M.K., Bonifaz, L., Steinman, R.M. \& Moran, T.M. Influenza virus-induced dendritic cell maturation is associated with the induction of strong $T$ cell immunity to a coadministered, mormally nonimmunogenic protein. J. Exp. Med. 198, 133-144 (2003).

67. Legge, K.L. \& Braciale, T.J. Accelerated migration of respiratory dendritic cells to the regional lymph nodes is limited to the early phase of pulmonary infection. Immunity 18, 265-277 (2003).

68. Legge, K.L. \& Braciale, T.J. Lymph node dendritic cells control CD8+ T cell responses through regulated FasL expression. Immunity 23, 649659 (2005).

69. Shortman, K. \& Naik, S.H. Steady-state and inflammatory dendritic-cell development. Nat. Rev. Immunol. 7, 19-30 (2007).

70. Belz, G.T. et al. Cutting edge: conventional CD8 alpha+ dendritic cells are generally involved in priming CTL immunity to viruses. J. Immunol. 172, 1996-2000 (2004).

71. Belz, G.T. et al. Distinct migrating and nonmigrating dendritic cell populations are involved in MHC class I-restricted antigen presentation after lung infection with virus. Proc. Natt. Acad. Sci. USA 101, 86708675 (2004).

72. Belz, G.T., Bedoui, S., Kupresanin, F., Carbone, F.R. \& Heath, W.R. Minimal activation of memory CD8 $+T$ cell by tissue-derived dendritic cells favors the stimulation of naive CD8+ T cells. Nat. Immunol. $\mathbf{8}$, 1060-1066 (2007).

73. Belz, G.T., Zhang, L., Lay, M.D., Kupresanin, F. \& Davenport, M.P. Killer T cells regulate antigen presentation for early expansion of memory, but not naive, CD8+ T cell. Proc. Natl. Acad. Sci. USA 104, 6341-6346 (2007).

74. Beaty, S.R., Rose, C.E. Jr \& Sung, S.S. Diverse and potent chemokine production by lung CD11bhi dendritic cells in homeostasis and in allergic lung inflammation. J. Immunol. 178, 1882-1895 (2007).

75. Rimmelzwaan, G.F., Baars, M.M., de Lijster, P., Fouchier, R.A. \& Osterhaus, A.D. Inhibition of influenza virus replication by nitric oxide. J. Virol. 73, 8880-8883 (1999).

76. Jewell, N.A. et al. Differential type I interferon induction by respiratory syncytial virus and influenza a virus in vivo. J. Virol. 81, 9790-9800 (2007).

77. Hao, X., Kim, T.S. \& Braciale, T.J. Differential response of respiratory dendritic cell subsets to influenza virus infection. J. Virol. 82, 4908-4919 (2008).

78. Hoeffel, G. et al. Antigen crosspresentation by human plasmacytoid dendritic cells. Immunity 27, 481-492 (2007).

79. Jego, G. et al. Plasmacytoid dendritic cells induce plasma cell differentiation through type I interferon and interleukin 6. Immunity 19, 225-234 (2003).

80. Sigurs, N. et al. Severe respiratory syncytial virus bronchiolitis in infancy and asthma and allergy at age 13. Am. J. Respir. Crit. Care Med. 171, 137-141 (2005).

81. Stein, R.T. et al. Respiratory syncytial virus in early life and risk of wheeze and allergy by age 13 years. Lancet 354, 541-545 (1999).

82. Schwarze, J., Hamelmann, E., Bradley, K.L., Takeda, K. \& Gelfand, E.W. Respiratory syncytial virus infection results in airway hyperresponsiveness and enhanced airway sensitization to allergen. J. Clin. Invest. 100, 226-233 (1997).

83. Wang, H., Peters, N. \& Schwarze, J. Plasmacytoid dendritic cells limit viral replication, pulmonary inflammation, and airway hyperresponsiveness in respiratory syncytial virus infection. J. Immunol. 177, 6263-6270 (2006).

84. Smit, J.J., Rudd, B.D. \& Lukacs, N.W. Plasmacytoid dendritic cells inhibit pulmonary immunopathology and promote clearance of respiratory syncytial virus. J. Exp. Med. 203, 1153-1159 (2006).

85. Smit, J.J. et al. The balance between plasmacytoid DC versus conventional $\mathrm{DC}$ determines pulmonary immunity to virus infections. PLOS ONE 3, e1720 (2008).

86. Grayson, M.H. et al. Induction of high-affinity lgE receptor on lung dendritic cells during viral infection leads to mucous cell metaplasia. J. Exp. Med. 204, 2759-2769 (2007).

87. Ostroukhova, M. et al. Tolerance induced by inhaled antigen involves CD4(+) T cells expressing membrane-bound TGF-beta and FOXP3. J. Clin. Invest. 114, 28-38 (2004).

88. Van Hove, C.L., Maes, T., Joos, G.F. \& Tournoy, K.G. Prolonged inhaled allergen exposure can induce persistent tolerance. Am. J. Respir. Cell Mol. Biol. 36, 573-584 (2007). 
89. Coombes, J.L. et al. A functionally specialized population of mucosal CD103+ DCs induces Foxp3+ regulatory T cells via a TGF-beta and retinoic acid-dependent mechanism. J. Exp. Med. 204, 1757-1764 (2007).

90. Reis e Sousa, C. Dendritic cells in a mature age. Nat. Rev. Immunol. 6, 476-483 (2006).

91. Akbari, O., DeKruyff, R.H. \& Umetsu, D.T. Pulmonary dendritic cells producing IL-10 mediate tolerance induced by respiratory exposure to antigen. Nat. Immunol. 2, 725-731 (2001).

92. Kohl, J. et al. A regulatory role for the C5a anaphylatoxin in type 2 immunity in asthma. J. Clin. Invest. 116, 783-796 (2006).

93. Ito, T. et al. Plasmacytoid dendritic cells prime IL-10-producing T regulatory cells by inducible costimulator ligand. J. Exp. Med. 204, 105115 (2007).

94. Martin, P. et al. Characterization of a new subpopulation of mouse CD8alpha(+) B220(+) dendritic cells endowed with type 1 interferon production capacity and tolerogenic potential. Blood 100, 383-390 (2002)

95. Ochando, J.C. et al. Alloantigen-presenting plasmacytoid dendritic cells mediate tolerance to vascularized grafts. Nat. Immunol. 7, 652-662 (2006).

96. Grohmann, U. et al. Reverse signaling through GITR ligand enables dexamethasone to activate IDO in allergy. Nat. Med. 13, 579-586 (2007).

97. Eisenbarth, S.C. et al. Lipopolysaccharide-enhanced, toll-like receptor 4-dependent T helper cell type 2 responses to inhaled antigen. J. Exp. Med. 196, 1645-1651 (2002).

98. Krishnamoorthy, N. et al. Activation of c-Kit in dendritic cells regulates T helper cell differentiation and allergic asthma. Nat. Med. 14, 565-573 (2008).

99. Hammad, H. \& Lambrecht, B.N. Dendritic cells and epithelial cells: linking innate and adaptive immunity in asthma. Nat. Rev. Immunol. 8, 193-204 (2008).

100. Idzko, M. et al. Local application of FTY720 to the lung abrogates experimental asthma by altering dendritic cell function. J. Clin. Invest. 116, 2935-2944 (2006).
101. Idzko, M. et al. Inhaled iloprost suppresses the cardinal features of asthma via inhibition of airway dendritic cell function. J. Clin. Invest. 117, 464-472 (2007).

102. Hammad, H. et al. Activation of the D prostanoid 1 receptor suppresses asthma by modulation of lung dendritic cell function and induction of regulatory T cells. J. Exp. Med. 204, 357-367 (2007).

103. Idzko, M. et al. Extracellular ATP triggers and maintains asthmatic airway inflammation by activating dendritic cells. Nat. Med. 13, 913-919 (2007).

104. van Rijt, L.S. et al. Allergen-induced accumulation of airway dendritic cells is supported by an increase in CD31 ${ }^{\text {hi }} \mathrm{Ly}-6 \mathrm{C}^{\text {neg }}$ hematopoietic precursors. Blood 100, 3663-3671 (2002).

105. Vermaelen, K. \& Pauwels, R. Accelerated airway dendritic cell maturation, trafficking, and elimination in a mouse model of asthma. Am. J. Respir. Cell Mol. Biol. 29, 405-409 (2003).

106. Upham, J.W., Denburg, J.A. \& O’Byrne, P.M. Rapid response of circulating myeloid dendritic cells to inhaled allergen in asthmatic subjects. Clin. Exp. Allergy 32, 818-823 (2002).

107. Van Rijt, L.S. et al. Essential role of dendritic cell CD80/CD86 costimulation in the induction, but not reactivation, of $\mathrm{TH} 2$ effector responses in a mouse model of asthma. J. Allergy Clin. Immunol. 114, 166-173 (2004).

108. Huh, J.C. et al. Bidirectional Interactions between Antigen-bearing Respiratory Tract Dendritic Cells (DCs) and T Cells Precede the Late Phase Reaction in Experimental Asthma: DC Activation Occurs in the Airway Mucosa but Not in the Lung Parenchyma. J. Exp. Med. 198, 19-30 (2003).

109. Vermaelen, K. et al. Matrix metalloproteinase-9-mediated dendritic cell recruitment into the airways is a critical step in mouse model of asthma J. Immunol. 171, 1016-1022 (2003).

110. Zhou, B. et al. Thymic stromal lymphopoietin as a key initiator of allergic airway inflammation in mice. Nat. Immunol. 6, 1047-1053 (2005). 